\title{
Isolation and characterization of draT mutants that have altered regulatory properties of dinitrogenase reductase ADP- ribosyltransferase in Rhodospirillum rubrum
}

\author{
Yaoping Zhang, ${ }^{1,2,3}$ Kitai Kim, ${ }^{2,3}$ Paul W. Ludden ${ }^{1,3}$ and Gary P. Roberts ${ }^{2,3}$
}

Author for correspondence: Gary P. Roberts. Tel: +1 608262 3567. Fax: +1 6082629865 .

e-mail: groberts@bact.wisc.edu

Departments of Biochemistry ${ }^{1}$ and Bacteriology ${ }^{2}$ and the Center for the Study of Nitrogen Fixation ${ }^{3}$, University of WisconsinMadison, Madison WI 53706, USA

\begin{abstract}
In Rhodospirillum rubrum, dinitrogenase reductase ADP-ribosyltransferase (DRAT) is responsible for the ADP-ribosylation of dinitrogenase reductase in response to the addition of $\mathrm{NH}_{4}^{+}$or removal from light, resulting in a decrease in nitrogenase activity. DRAT is itself subject to post-translational regulation; to investigate the mechanism for the regulation of DRAT activity, random PCR mutagenesis of draT (encoding DRAT) was performed and mutants with altered DRAT regulation were screened. Two mutants (with substitutions of K103E and N248D) were obtained in which DRAT showed activity under conditions where wild-type DRAT (DRAT-WT) did not. These mutants showed lower nitrogenase activity and a higher degree of ADP-ribosylation of dinitrogenase reductase under $\mathbf{N}_{\mathbf{2}}$-fixing conditions than was seen in a wild-type control strain. DRATK103E was overexpressed and purified. DRAT-K103E displayed a much weaker affinity for an Affi-gel Blue matrix than did DRAT-WT, suggestive of a fairly striking biochemical change. However, there was no significant difference in kinetic constants, such as $K_{m}$ for NAD and $V_{\text {max }}$, between DRAT-K103E and DRATWT. Like DRAT-WT, DRAT-K103E also modified reduced dinitrogenase reductase poorly. The biochemical properties of these variants are rationalized with respect to their behaviour in vivo.
\end{abstract}

Keywords: nitrogen fixation, regulation, ADP-ribosylation, random PCR mutagenesis

\section{INTRODUCTION}

In the photosynthetic bacterium Rhodospirillum rubrum, the regulation of nitrogenase activity involves the reversible ADP-ribosylation of dinitrogenase reductase (Ludden \& Roberts, 1989). Two enzymes that perform this regulation have been characterized. As shown in the model in Fig. 1, dinitrogenase reductase ADP-ribosyltransferase (referred to as DRAT) carries out the transfer of the ADP-ribose from NAD to the Arg-101 residue of one subunit of the dinitrogenase reductase dimer, resulting in inactivation of that enzyme. Dinitrogenase reductase activating glycohydrolase (referred to as DRAG) removes the ADP-ribose from the covalently modified dinitrogenase reductase, restoring its activity.

Abbreviations: DRAG, dinitrogenase reductase activating glycohydrolase; DRAT, dinitrogenase reductase ADP-ribosyltransferase; DRATWT, DRAT from wild-type.
The DRAT/DRAG regulatory approach has also been found in other nitrogen-fixing bacteria, such as the phototroph Rhodobacter capsulatus (Jouanneau et al., 1989; Masepohl et al., 1993; Pierrard et al., 1993) and the non-photosynthetic Azospirillum brasilense and Azospirillum lipoferum (Fu et al., 1989; Zhang et al., 1992). In $R$. rubrum and $R b$. capsulatus, this system responds to fixed nitrogen and to darkness (Kanemoto \& Ludden, 1984; Liang et al., 1991; Masepohl et al., 1993; Pierrard et al., 1993); in A. brasilense it responds to ammonium and anaerobiosis ( $\mathrm{Fu}$ et al., 1989; Hartmann \& Burris, 1987; Hartmann et al., 1986; Zhang et al., 1992, 1993). The draTG genes, which encode DRAT and DRAG, have been cloned and sequenced from each of these bacteria (Fitzmaurice et al., 1989; Fu et al., 1990; Inoue et al., 1996; Ma \& Li, 1997; Masepohl et al., 1993; Zhang et al., 1992); the sequence comparisons showed extensive similarity, with some particularly conserved regions (Masepohl et al., 1993). 


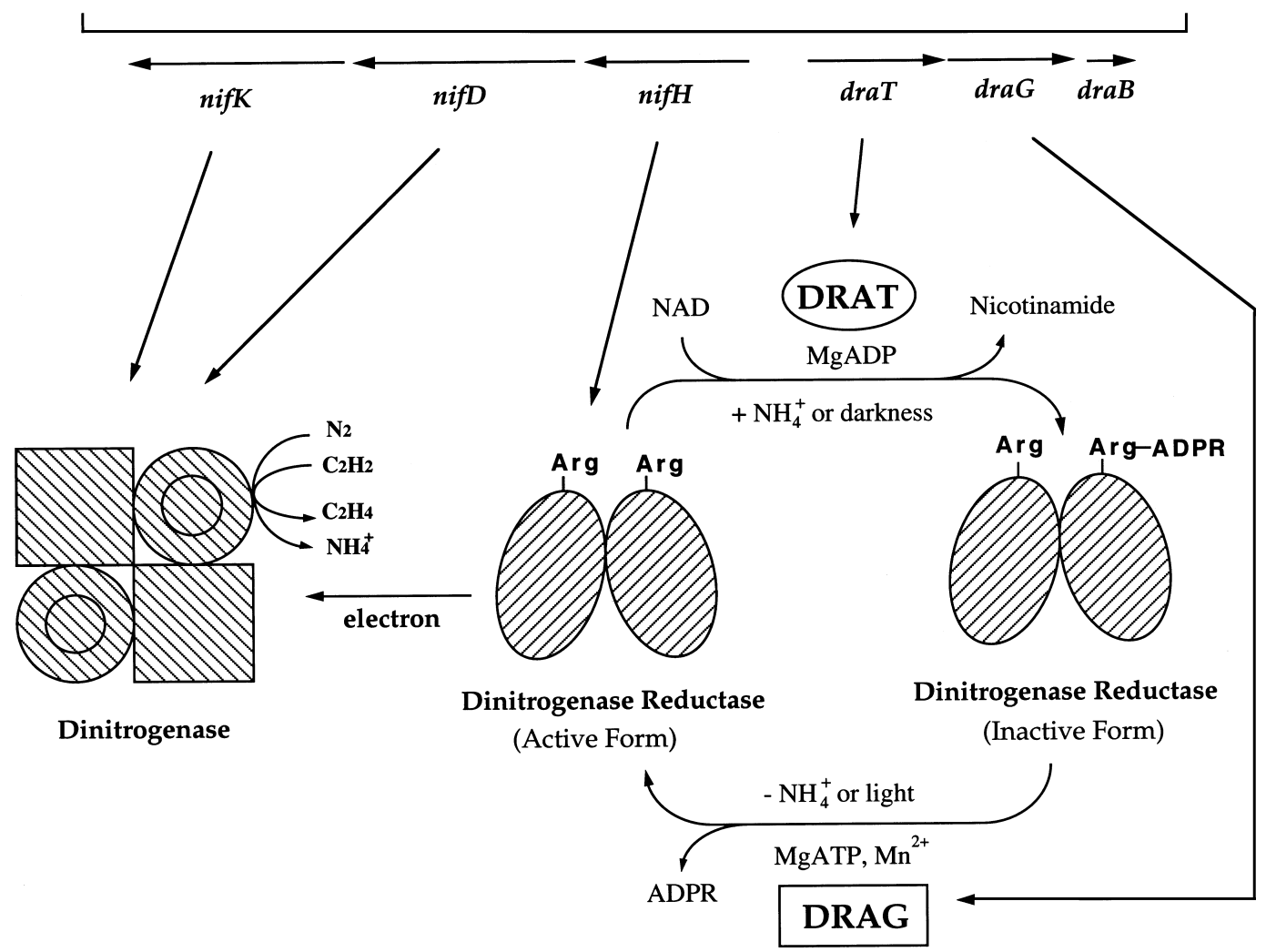

Fig. 1. Model for the regulation of dinitrogenase reductase in $R$. rubrum by ADP-ribosylation.

Through biochemical and genetic analysis, it has become clear that the activities of DRAT and DRAG are themselves subject to post-translational regulation (Zhang et al., 1997). Under nitrogen-fixing conditions, DRAT appears to be inactive. Following treatment with a stimulus that is 'negative' for nitrogen fixation, such as $\mathrm{NH}_{4}^{+}$or darkness (energy depletion), DRAT is activated, resulting in the modification of dinitrogenase reductase and the loss of nitrogenase activity. However, DRAT activation is only transient and DRAT becomes inactive again even in the continued presence of the negative stimulus (Zhang et al., 1993). In contrast, DRAG is active under nitrogen-fixing conditions and is inactivated by the negative stimulus. Unlike the regulation of DRAT, however, the regulation of DRAG is not transient, but reflects the current physiological status (Kanemoto \& Ludden, 1984). Following the negative stimulus, DRAG becomes inactive and remains inactive until the removal of the negative stimulus. DRAG then reactivates dinitrogenase reductase by cleavage of the ADP-ribose group.

Details of the mechanisms for the regulation of DRAT and DRAG activities themselves are still unknown. Both DRAT and DRAG display substantial activity in vitro either when purified or in crude extracts of cells grown under various conditions (Lowery \& Ludden, 1989; Triplett et al., 1982). This loss of regulation upon cell breakage suggests that DRAT and DRAG might be regulated by the inhibition of their activities through loosely binding negative effector(s). Recently, it was found that the redox states of dinitrogenase reductase affect its ability to serve as a substrate for DRAT and DRAG, both in vitro and in vivo (Halbleib et al., 2000). DRAT can only modify oxidized dinitrogenase reductase and DRAG only removes the ADP-ribosyl group from reduced dinitrogenase reductase. However, whilst the redox state of dinitrogenase reductase probably plays an important role in the regulation of DRAT and DRAG activities, it cannot be the only type of regulation. As mentioned above, DRAT and DRAG are not always regulated in a coordinated way and both proteins can be inactive under some conditions; thus, the regulation of DRAT and DRAG activities must involve additional factors.

To investigate the mechanism of DRAT regulation, we employed random PCR mutagenesis of draT from $R$. rubrum and screened for mutants with regulationaltered DRAT. Our success in finding such mutants with relative ease is most easily rationalized on the basis of a model in which these DRAT variants have been damaged at a site where a negative effector binds. This result provides support for the hypothesis of loosebinding negative effectors regulating DRAT activity in vivo.

\section{METHODS}

Bacterial strains and plasmids. The strains of R. rubrum and the plasmids used in this study are listed in Table 1. 
Table 1. Rhodospirillum strains and plasmids

\begin{tabular}{|c|c|c|}
\hline Strain/plasmid & Relevant genotype and description & Reference \\
\hline \multicolumn{3}{|l|}{ Strains } \\
\hline UR2 & Wild-type & Kanemoto \& Ludden (1984) \\
\hline UR212 & draT2::kan mutant & Liang et al. (1991) \\
\hline UR462 & UR212 with pYPZ163 (Pdra-draT) & This study \\
\hline UR472 & $\Delta d r a T G B 10:: k a n$ mutant & This study \\
\hline UR483 & UR472 with pYPZ163 (Pdra-draT) & This study \\
\hline UR506 & UR2 with pYPZ163 (Pdra-draT) & This study \\
\hline UR508 & UR2 with pYPZ170 (Pdra-draT16, K103E) & This study \\
\hline UR511 & UR212 with pYPZ170 (Pdra-draT16, K103E) & This study \\
\hline UR578 & UR472 with pYPZ174 (Pnif-draT16, K103E) & This study \\
\hline UR581 & UR2 with pYPZ179 (Pdra-draT17, N248D) & This study \\
\hline UR582 & UR212 with pYPZ179 (Pdra-draT17, N248D) & This study \\
\hline UR583 & pYPZ173 (draT16, K103E) was integrated into the chromosome of UR472 & This study \\
\hline UR587 & UR472 with pYPZ183 (Pnif-draT16GB, K103E) & This study \\
\hline UR589 & pYPZ187 (wild-type draT) was integrated into the chromosome of UR472 & This study \\
\hline UR610 & pYPZ190 (draT17, N248D) was integrated into the chromosome of UR472 & This study \\
\hline UR619 & pYPZ191 (draT18, N46D) was integrated into the chromosome of UR472 & This study \\
\hline \multicolumn{3}{|c|}{ 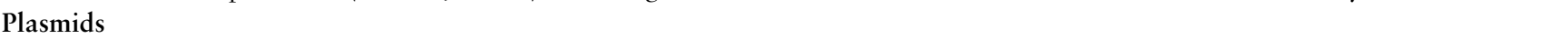 } \\
\hline pUX113 & $\begin{array}{l}\text { Overexpression plasmid of } d r a T \text { under the control of the nifH promoter on } \\
\text { pRK404 (Pnif-draT) }\end{array}$ & Grunwald et al. (1995) \\
\hline pYPZ148 & $3 \cdot 3 \mathrm{~kb}$ draTGB was cloned into pUC19 & This study \\
\hline pYPZ162 & $\begin{array}{l}2 \cdot 3 \mathrm{~kb} \text { draTGB', together with aacC1 gene (encoding } \mathrm{Gm}^{\mathrm{R}} \text { ) from pUCGM, was } \\
\text { cloned into pRK404 (Pdra-draTG) }\end{array}$ & This study \\
\hline pYPZ163 & $\begin{array}{l}0 \cdot 8 \mathrm{~kb} \text { EcoRI fragment containing } d r a G^{\prime} B^{\prime} \text { was deleted from pYPZ162 } \\
(\mathrm{P} d r a-d r a T)\end{array}$ & This study \\
\hline pYPZ165 & $1 \mathrm{~kb} B a m \mathrm{HI}-E c o \mathrm{RV}$ fragment containing $d r a T$ was cloned into pBSKS $(-)$ & This study \\
\hline pYPZ170 & Similar to pYPZ163, but contains Pdra-draT16 (K103E) & This study \\
\hline pYPZ173 & Similar to pYPZ187, but contains Pdra-draT16 (K103E) & This study \\
\hline pYPZ174 & Similar to pUX113, but contains Pnif-draT16 (K103E) & This study \\
\hline pYPZ179 & Similar to pYPZ163, but contains Pdra-draT17 (N248D) & This study \\
\hline pYPZ183 & $1 \cdot 2 \mathrm{~kb} d r a G^{\prime} B$ was cloned into pYPZ174 (Pnif-draT16GB, K103E) & This study \\
\hline pYPZ187 & $\begin{array}{l}\text { Pdra-draT, together with aacC1 gene (encoding } \mathrm{Gm}^{\mathrm{R}} \text { ) from pUCGM, was cloned } \\
\text { into pSUP202, a suicide vector for } R \text {. rubrum }\end{array}$ & This study \\
\hline pYPZ190 & Similar to pYPZ187, but contains Pdra-draT17 (N248D) & This study \\
\hline pYPZ191 & Similar to pYPZ187, but contains Pdra-draT18 (N46D) & This study \\
\hline
\end{tabular}

Growth conditions and whole-cell nitrogenase activity assay. The method of derepression for nitrogenase in $R$. rubrum has been described previously (Kanemoto \& Ludden, 1984), but has been modified for simplicity. R. rubrum was first grown in rich (SMN) medium (Fitzmaurice et al., 1989), then directly inoculated into malate-glutamate (MG) medium (Lehman \& Roberts, 1991) with 60-fold dilution. After evacuation of the gas space and flushing with argon, cultures were derepressed for nitrogenase under light for $2 \mathrm{~d}$. The whole-cell nitrogenase activity assay and the darkness $/ \mathrm{NH}_{4} \mathrm{Cl}$ treatments have been described previously (Zhang et al., 1995a). For purification of DRAT and dinitrogenase reductase, $R$. rubrum was grown in 22-litre carboys. The MG medium was supplemented with fructose $\left(5 \mathrm{~g} \mathrm{l}^{-1}\right)$ to increase cell yields.

Construction of $\boldsymbol{R}$. rubrum draTG deletion mutant. To construct a draTG deletion mutant, a $3.3 \mathrm{~kb}$ Pst I fragment containing draTGB (Fitzmaurice et al., 1989) was subcloned into pUC19, yielding pYPZ148. draB is an open reading frame (ORF) immediately downstream of $d r a G$ in R. rubrum, A. brasilense and A. lipoferum (Fitzmaurice et al., 1989; Inoue et al., 1996; Ma \& Li, 1997; Zhang et al., 1992) and loss-offunction mutations in this gene cause a small decrease in DRAG activity in R. rubrum (Liang et al., 1991). In this report, we have decided to give this ORF a $d r a$ gene designation $(d r a B)$ both because of the mutation phenotype and because of the apparent co-transcription of $d r a B$ with $d r a T G$ (D. P. Lies \& G. P. Roberts, unpublished data). pYPZ148 was digested with $A$ flIII and BstBI and this deleted part of draT, all of $d r a G$ and part of $d r a B$. After treatment with mung bean nuclease to create blunt ends, this fragment was ligated with a $1.4 \mathrm{~kb}$ HincII fragment containing the $\mathrm{Km}^{\mathrm{R}}$ cassette gene from pUC4K (Vieira \& Messing, 1982), so that the deleted region of draT'draGdraB' was replaced by the $\mathrm{Km}^{\mathrm{R}}$ cassette. The deletion/insertion region was subcloned into pSUP202 (Simon et al., 1983) and conjugated into the $R$. rubrum wild-type, as described previously (Liang et al., 1991). pSUP202 is unable to replicate in $R$. rubrum and $\mathrm{Sm}^{\mathrm{R}} \mathrm{Km}^{\mathrm{R}}$ colonies were selected and replica-printed to screen for $\mathrm{Cm}^{\mathrm{S}}\left(\mathrm{Cm}^{\mathrm{R}}\right.$ is encoded by the vector sequence) colonies resulting from a double-crossover recombination event. The mutation eliminates functional 
DRAT and DRAG and was designated $\triangle$ draTGB10::kan. The strain was designated UR472 and was used thereafter as a host strain to screen and analyse $d r a T$ mutations.

Integration of draT alleles into the chromosome of UR472 ( $\Delta$ draTGB10::kan). The various draT alleles were cloned into pSUP202, then transferred into $R$. rubrum UR472. Transconjugants with a single crossover were selected $\left(\mathrm{Sm}^{\mathrm{R}} \mathrm{Tc}^{\mathrm{R}}\right)$, so that a single copy of $d r a T$ was integrated into the chromosome, but no functional allele of $d r a G$ was present.

Random PCR mutagenesis. The protocol for random PCR mutagenesis was based on the methods described previously (Leung et al., 1989; Vogel \& Das, 1994). To increase the mutation frequency, $0.5 \mathrm{mM} \mathrm{MnCl}{ }_{2}$ and high concentrations of dGTP, dCTP and dTTP $(1 \mathrm{mM})$, as well as a low concentration of dATP $(0 \cdot 2 \mathrm{mM})$, were used. The cycle profile was as follows: $45 \mathrm{~s}$ at $94{ }^{\circ} \mathrm{C}, 1 \mathrm{~min}$ at $42{ }^{\circ} \mathrm{C}$ and $2.5 \mathrm{~min}$ at $72{ }^{\circ} \mathrm{C}$, for 30 cycles. Taq DNA polymerase from Thermus aquaticus (Fisher) was used in the reaction, as it supports a high mutation frequency (Keohavong \& Thilly, 1989; Lundberg et al., 1991).

Three plasmids, pYPZ162, pYPZ163 and pYPZ165, were constructed for random PCR mutagenesis and screening. A $1 \mathrm{~kb} \mathrm{BamHI-EcoRI} \mathrm{fragment} \mathrm{of} R$. rubrum draT was subcloned into pBSKS $(-)$ (Stratagene), yielding pYPZ165. This plasmid was used as a template for the PCR reaction. A $2 \cdot 3 \mathrm{~kb}$ fragment containing $R$. rubrum nif $H^{\prime} d r a T G B^{\prime}$ was cloned into pRK404 (Ditta et al., 1985), and an aacC1 gene from pUCGM encoding gentamicin acetyltransferase-3-1 $\left(\mathrm{Gm}^{\mathrm{R}}\right)$ (Schweizer, 1993) was inserted at the $3^{\prime}$-end of the nifH' gene, yielding pYPZ162. A $0 \cdot 8 \mathrm{~kb}$ EcoRI fragment containing $d r a G^{\prime} B^{\prime}$ was deleted from pYPZ162, yielding pYPZ163. After PCR amplification with pYPZ165 as a template, DNA was precipitated with ethanol and digested with BamHI and EcoRI, then ligated with pYPZ163 which had been digested with the same restriction enzymes to delete wild-type draT. The ligation mixture was transformed into Escherichia coli $\mathrm{DH} 5 \alpha$ to generate a $d r a \mathrm{~T}$ mutant library.

Screening of drat mutants. Plasmids from the draT mutant library were transferred into R. rubrum strain UR212 or strain UR472 as described previously (Grunwald et al., 1995). $\mathrm{Sm}^{\mathrm{R}}$ $\mathrm{Tc}^{\mathrm{R}} \mathrm{Gm}^{\mathrm{R}}$ transconjugants of $R$. rubrum were grown in $\mathrm{SMN}$, then inoculated into nitrogen-free liquid medium at a 200 -fold dilution or diluted and plated on nitrogen-free medium. This medium was similar to MN medium (Lehman \& Roberts, 1991), except that $\mathrm{NH}_{4} \mathrm{Cl}$ was omitted. Cells were grown anaerobically in nitrogen-free medium containing $10 \mu \mathrm{g}$ gentamicin $\mathrm{ml}^{-1}$ under a dark/light regimen, with 90 min light and 30 min dark. This increased the difference in the growth rate between the mutants and the wild-type.

DNA sequencing analysis. DNA sequences were determined using the ABI PRISM Dye Terminator Cycle Sequencing Kit (Perkin-Elmer). Sequencing data were analysed with DNASTAR software programs (DNASTAR).

DRAT overexpression. The overexpression plasmid (pUX113) for wild-type $d r a T$ was constructed previously (Grunwald et al., 1995). For overexpression of DRAT-K103E, the BbsIBsaI fragment, containing mutated draT16 (K103E) from pYPZ170, was cloned into pUX113 to replace the $d r a^{+}$region, yielding pYPZ174.

Protein purification. The purification of dinitrogenase reductases and DRAT has been reported previously (Grunwald \& Ludden, 1997; Lowery \& Ludden, 1988). On the Affi-gel column, DRAT-K103E showed substantially weaker binding than did DRAT-WT, eluting with $150 \mathrm{mM} \mathrm{NaCl}$ and $1 \mathrm{mM}$ ADP. After the Affi-gel Blue column, DRAT-K103E was bound to a Phenyl Sepharose column (Pharmacia Biotech) and eluted with $50 \%$ ethylene glycol.

Protein immunoblotting. A trichloroacetic acid precipitation method and the protein immunoblotting procedure have been described previously (Zhang et al., 1993). ECL Western blotting detection reagents (Amersham) were used for detection.

In vitro DRAT activity assay. NAD labelled with ${ }^{32} \mathrm{P}$ was used as a donor of ADP-ribose for the in vitro DRAT activity assay, as described previously (Grunwald et al., 1995; Grunwald \& Ludden, 1997; Lowery \& Ludden, 1988). DRAT activity is expressed as nmol ADP-ribose transferred per min per mg protein.

\section{RESULTS}

\section{Random PCR mutagenesis and screening of draT mutants from $R$. rubrum}

It has been our working hypothesis that DRAT activity is regulated through dissociable negative effector(s). We tested this hypothesis by seeking DRAT variants that have lost the site at which such effectors would bind; this should result in strains with 'constitutive' DRAT activity. Because of the lack of easy selection/screening methods for such regulatory mutants, it was important to mutagenize draT efficiently so that the desired mutants would exist at a detectable frequency. We therefore employed random PCR mutagenesis on draT.

First, we developed a method of screening for the desired draT mutants, based on the expected difference between wild-type and draT mutants in terms of their nitrogenase activity and growth rates in nitrogen-free medium (Table 2). Strain UR212 (draT::kan) was used as the host strain for screening the plasmid-borne mutants. Because of the kan insertion in draT and its polar effect on $d r a G$, UR212 has no DRAT and has a low DRAG activity (less than $5 \%$ of wild-type DRAG activity) (Liang et al., 1991). This low DRAG activity has three advantages in the screen. First, unlike wild-type DRAG levels, this low DRAG activity should provide less competition for the desired 'constitutively' active DRAT, so nitrogenase activity in these mutants should be very low. Second, this low DRAG activity supported high initial nitrogenase activity in the presence of multicopy draT $T^{+}$. Although most of the DRAT from this multicopy plasmid was still regulated normally and was in an inactive form under $\mathrm{N}_{2}$-fixing conditions, a low background level of DRAT activity was present, which resulted in the dramatic decrease in nitrogenase activity and the modification of dinitrogenase reductase when no DRAG was present. Such a low nitrogenase activity with plasmid-borne DRAT-WT would make it difficult to screen for 'constitutively' active DRAT mutants that cause even lower nitrogenase activity. However, the low DRAG activity in UR212 compensated for the leaky DRAT-WT activity and supported a higher level of nitrogenase activity. Third, this low DRAG activity supported the recovery of 
Table 2. Summary of the screening of $d r a T$ mutants and their expected phenotypes in nitrogen-free medium with a light/dark-cycle regimen

Strain UR212 (draT::kan) was used as the host strain for screening the plasmid-borne mutants. Because of the kan insertion in draT and its polar effect on $d r a G$, UR212 has no DRAT and has low DRAG activity (less than $5 \%$ of wild-type DRAG activity).

\begin{tabular}{|c|c|c|c|c|}
\hline $\begin{array}{l}\text { Type of DRAT mutant } \\
\text { on the plasmid in the } \\
\text { UR212 background }\end{array}$ & $\begin{array}{c}\text { Nitrogenase activity } \\
\text { (modification of } \\
\text { dinitrogenase reductase) } \\
\text { under light }\end{array}$ & $\begin{array}{l}\text { Nitrogenase activity } \\
\text { under light/dark } \\
\text { regimen }\end{array}$ & $\begin{array}{l}\text { Growth rate in } \\
\mathrm{N} \text {-free medium }\end{array}$ & $\begin{array}{c}\text { No. of } \\
\text { mutants found }\end{array}$ \\
\hline $\begin{array}{l}\text { No mutation or silent } \\
\text { mutation (wild-type } \\
\text { DRAT) }\end{array}$ & $\begin{array}{l}\text { Medium activity (some } \\
\text { modification) }\end{array}$ & $\begin{array}{l}\text { Loses activity and } \\
\text { recovers }\end{array}$ & Medium & NA \\
\hline $\begin{array}{l}\text { 'Constitutively' active } \\
\text { DRAT }\end{array}$ & $\begin{array}{l}\text { Low activity (complete } \\
\text { modification) }\end{array}$ & $\begin{array}{l}\text { Loses activity, but does } \\
\text { not recover }\end{array}$ & Slow & 2 \\
\hline Loss-of-function DRAT & $\begin{array}{l}\text { High activity (no } \\
\text { modification) }\end{array}$ & Remains active & Fast & $>50$ \\
\hline
\end{tabular}

NA, Not applicable.

nitrogenase activity after cells were shifted from dark to light. This increased the difference in growth rate between the mutants with 'constitutively' active DRAT and the wild-type under the light/dark cycle regimen.

Different $d r a T$ mutants were expected to show different growth rates under these conditions (Table 2). Strain UR212 with wild-type draT on the plasmid (strain UR462) shows a medium level of nitrogenase activity and shows moderate growth in nitrogen-free medium under dark/light shift conditions, because even the low DRAG activity of this strain supports moderate initial nitrogenase activity and allows the recovery of nitrogenase activity after each shift from darkness to light. draT mutants that have lost catalytic activity (referred to as the loss-of-function mutants) have a faster rate of growth, since they have higher initial nitrogenase activity and retain their nitrogenase reductase in an active form during each shift to darkness. In contrast, if the UR212 derivative contained a 'constitutively' active DRAT, this strain would have a very poor growth rate, because this 'constitutively' active DRAT could compete with the low DRAG activity, resulting in a low nitrogenase activity regardless of illumination conditions.

Although mutants with 'constitutively' active DRAT are the most interesting ones, loss-of-function mutants were also analysed; they were used to estimate mutation frequency and to determine whether these mutants clustered in specific domains of DRAT. A different $R$. rubrum strain, UR472 ( $\Delta$ draTGB), was used as the host strain for screening this type of mutant. Unlike DRAG activity in UR212 (draT::kan), which is low, DRAG activity in UR472 is completely eliminated. When multicopy draT was introduced into UR472 (creating UR483; Table 3), the strain showed a very low nitrogenase activity and grew slowly in nitrogen-free medium. A mutant with a loss-of-function mutation in draT would grow faster under these conditons.
After random PCR mutagenesis, draT mutants were screened in nitrogen-free medium. After a $4-5 \mathrm{~d}$ incubation under a dark/light regimen, colonies or liquid cultures with faster or slower growth rates than that of the wild-type control (UR462 or UR483) were picked.

\section{Characterization of draT mutants}

Approximately 1000 mutagenized plasmids were screened in the background of UR472 ( $\Delta$ draTGB::kan); this indicated that approximately $8 \%$ of the clones represented loss-of-function draT mutants, and some were analysed further. Another 1000 mutagenized plasmids were screened in the UR212 (draT::kan) background, which allowed detection of mutants with altered DRAT regulation, and two mutants (UR542 and UR543) were found to grow poorly under these selection conditions, suggesting that they had 'constitutively' active DRAT. Single colonies were purified from both classes of mutants, and the nitrogenase activity and its regulation were reanalysed. As shown in Table 3, most of the putative loss-of-function mutants showed a higher initial nitrogenase activity than did the control strains (UR483 or UR462), resulting in a fast growth rate in nitrogen-free medium under screening conditions. In these mutants, the regulation of nitrogenase activity in response to darkness was partially or completely abolished. Most of these mutants showed a high residual nitrogenase activity (30-100\% of the initial activity before treatment) after a shift to darkness for $60 \mathrm{~min}$, whereas a very low residual nitrogenase activity $(5 \%)$ was seen in wild-type control strains (UR462 and UR483). One mutant (UR537) showed a normal response to dark treatment, but with high initial nitrogenase activity. This mutant is characterized below. Western blotting with antibodies to DRAT revealed that, out of 44 loss-of-function mutants, 30 mutants accumulated little or no DRAT protein, whilst 14 mutants accumulated levels of DRAT protein comparable to those in UR462 (data not shown). Two 
Table 3. Summary of draT mutants obtained from random PCR mutagenesis

\begin{tabular}{|c|c|c|c|c|}
\hline $\begin{array}{l}\text { R. rubrum } \\
\text { strain }\end{array}$ & Background strain & $\begin{array}{l}\text { Mutations in } \\
\text { draT on plasmid }\end{array}$ & $\begin{array}{c}\text { Initial } \\
\text { nitrogenase } \\
\text { activity* }\end{array}$ & $\begin{array}{l}\text { Darkness } \\
\text { response } †\end{array}$ \\
\hline UR483 & UR472 ( $\Delta d r a T G B:: k a n)$ & Wild-type & 30 & Normal \\
\hline UR525 & UR472 ( $\Delta d r a T G B:: k a n)$ & M52T & 400 & None \\
\hline UR528 & UR472 ( $\Delta d r a T G B:: k a n)$ & F74L & 300 & Partial \\
\hline UR529 & UR472 ( $\Delta d r a T G B:: k a n)$ & Y69C, L263P & 600 & None \\
\hline UR530 & UR472 ( $\Delta d r a T G B:: k a n)$ & L189P, M201V & 600 & None \\
\hline UR531 & UR472 ( $\Delta d r a T G B:: k a n)$ & $\mathrm{I} 245 \mathrm{~V}, \mathrm{~L} 276 \mathrm{P}$ & 600 & None \\
\hline UR532 & UR472 ( $\Delta d r a T G B:: k a n)$ & L76P, F230S, S274S & 300 & None \\
\hline UR534 & UR472 ( $\Delta d r a T G B:: k a n)$ & L250P & 250 & Partial \\
\hline UR535 & UR472 ( $\Delta d r a T G B:: k a n)$ & W144R & 400 & Partial \\
\hline UR537 & UR472 ( $\Delta d r a T G B:: k a n)$ & N46D & 450 & Normal \\
\hline UR462 & UR212 (draT::kan) & Wild-type & 350 & Normal \\
\hline UR542 & UR212 (draT::kan) & K103E, T237A & 40 & Normal \\
\hline UR543 & UR212 (draT::kan) & $\mathrm{N} 248 \mathrm{D}$ and promoter & 100 & Normal \\
\hline UR546 & UR212 (draT::kan) & Y169C & 700 & Partial \\
\hline UR549 & UR212 (draT::kan) & M73V, Y107N, Q164R & 900 & None \\
\hline UR550 & UR212 (draT::kan) & G19E, F74L, E79E & 500 & None \\
\hline UR551 & UR212 (draT::kan) & G266S & 800 & None \\
\hline UR552 & UR212 (draT::kan) & Y190C, Y257stop & 800 & None \\
\hline
\end{tabular}

* Nitrogenase activity is expressed as nmol ethylene produced per $\mathrm{h}$ per $\mathrm{ml}$ of cells at $\mathrm{OD}_{600} 1$.

† To test the response to darkness, cells were shifted from light to darkness for $60 \mathrm{~min}$ and then the nitrogenase activity was assayed. The response was scored as follows : none, $80-100 \%$ of the initial nitrogenase activity remained under conditions of darkness; partial, 30-80 \% of the initial nitrogenase activity remained; normal, $5-20 \%$ of the initial nitrogenase activity remained.

Table 4. Comparison of nitrogenase activity from strains with plasmids containing wild-type or altered draT

\begin{tabular}{|c|c|c|c|c|c|c|}
\hline \multirow[t]{2}{*}{ Strain } & \multirow{2}{*}{$\begin{array}{l}\text { Chromosomal } \\
\text { genotype }\end{array}$} & \multirow{2}{*}{$\begin{array}{l}\text { Substitution(s) in } \\
\text { draT on pRK } 404\end{array}$} & \multicolumn{3}{|c|}{ Estimated level of protein accumulation* } & \multirow{2}{*}{$\begin{array}{c}\text { In vivo } \\
\text { nitrogenase } \\
\text { activity } \|\end{array}$} \\
\hline & & & $\mathrm{DRAT}^{\mathrm{wt}}+$ & $\operatorname{DRAT}^{\mathrm{c}} \neq$ & DRAG $\mathbb{S}$ & \\
\hline UR2 (wild-type) & $d r a^{+}$ & & 1 & 0 & 1 & 800 \\
\hline UR462 & draT2::kan & Wild-type draT & 10 & 0 & $<0 \cdot 05$ & 360 \\
\hline UR511 & draT2::kan & $\mathrm{K} 103 \mathrm{E}($ draT16) & 0 & 10 & $<0 \cdot 05$ & 60 \\
\hline UR582 & draT2::kan & $\mathrm{N} 248 \mathrm{D}$ (draT17) & 0 & 10 & $<0 \cdot 05$ & 120 \\
\hline UR506 & $d r a^{+}$ & Wild-type $d r a T$ & 11 & 0 & 1 & 560 \\
\hline UR508 & $d r a^{+}$ & $\mathrm{K} 103 \mathrm{E}(\operatorname{draT16})$ & 1 & 10 & 1 & 250 \\
\hline UR581 & $d r a^{+}$ & $\mathrm{N} 248 \mathrm{D}($ draT17) & 1 & 10 & 1 & 600 \\
\hline
\end{tabular}

* The level of DRAT and DRAG is 1 for the wild-type and 10 for strains containing multiple Pdra-draT on pRK404, which is at about 10 copies per cell.

† Wild-type DRAT.

$\ddagger$ Constitutively active DRAT.

\Low DRAG activity (less than $5 \%$ of the wild-type activity) was seen in the $d r a T:$ : $k a n$ mutant, because the $\mathrm{Km}^{\mathrm{R}}$ insertion has a polar effect on draG (Liang et al., 1991).

$\|$ Nitrogenase activity is expressed as nmol ethylene produced per h per $\mathrm{ml}$ of cells at $\mathrm{OD}_{600} 1$.

poorly growing strains (UR542 and UR543) showed very low nitrogenase activity under $\mathrm{N}_{2}$-fixing conditions, and had a level of DRAT protein accumulation comparable to that of UR462, consistent with the presence of 'constitutively' active DRAT variants.
Plasmids were isolated from 16 mutants with a normal level of DRAT: 14 were loss-of-function mutants and two were 'constitutively' active DRAT variants. In each case, the entire mutated draT region was sequenced: seven plasmids had a single mutation, six plasmids had 


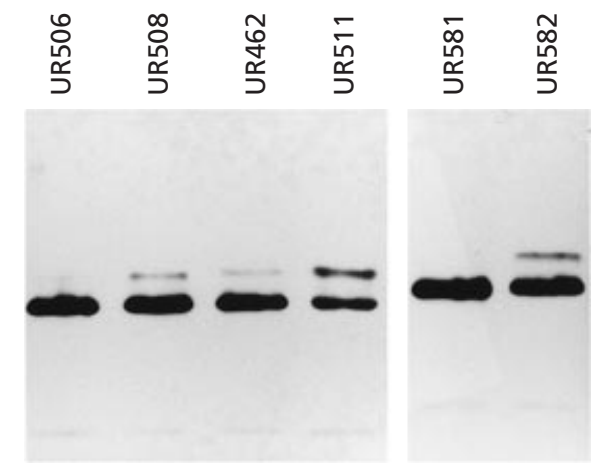

Fig. 2. Immunoblots of dinitrogenase reductase from strains grown under nitrogen-fixing conditions and containing plasmids encoding DRAT-WT (UR462 and UR506), DRAT-K103E (UR511 and UR508) and DRAT-N248D (UR582 and UR581). The parental strain of UR462, UR511 and UR582 is UR212 (draT2::kan), which has low DRAG activity. The parental strain of UR506, UR508 and UR581 is wild-type UR2.

two mutations, and three plasmids had three mutations. Most $(89 \%)$ of the mutations were transitions. These draT mutants are summarized in Table 3. DNA sequence analysis showed no clustering of the mutations in the loss-of-function mutants, and no alleles had identical sequences. UR537 has a mutation in draT causing an N46D substitution. Both of the mutants with 'constitutively' active DRAT had two mutations in draT: UR542 had mutations causing K103E and T237A; UR543 had a mutation causing N248D and another mutation located in the promoter region of $d r a T$.

\section{Further characterization of the mutants with altered regulation of DRAT activity}

To identify which substitutions caused the altered regulation of DRAT activity, a smaller fragment of draT, containing only a single substitution of T237A, K103E or N248D, was subcloned into pYPZ163, yielding pYPZ169 (T237A), pYPZ170 (K103E) and pYPZ179 (N248D). These plasmids were transferred into different R. rubrum strains for further analysis. For the following experiments, we created strains with different ratios of DRAT and DRAG to verify the presence of 'constitutive' DRAT activity in these DRAT variants.

In the UR212 (draT2::kan, with a low DRAG activity) background, strains with plasmid-borne DRAT-T237A (UR544) showed a high level of nitrogenase activity under $\mathrm{N}_{2}$-fixing conditions, like the strain with DRATWT (data not shown). This indicated that the T237A is not the cause of the 'constitutive' DRAT activity. However, strains with plasmid-borne DRAT-K103E (UR511) or DRAT-N248D (UR582) showed 20-35\% of the nitrogenase activity of the wild-type control (UR462) under $\mathrm{N}_{2}$-fixing conditions (Table 4). Modification of dinitrogenase reductase was monitored by Western blotting (Fig. 2). Since only one subunit of dinitrogenase reductase is modified and the ADP-ribosylation slows the migration of that subunit, two bands are seen for the inactive form. Consistent with this, a high degree of modification of dinitrogenase reductase was found in UR511 (DRAT-K103E) and in UR582 (DRAT-N248D), compared with the wild-type control (UR462) under $\mathrm{N}_{2}$ fixing conditions. These results indicate that these altered DRAT proteins are significantly active under $\mathrm{N}_{2}$-fixing conditions. These DRAT variants, when expressed from the plasmid at levels approximately 10 times that of the wild-type, can compete effectively with the low level of DRAG in this strain background to modify dinitrogenase reductase. However, we have no way of accurately quantifying in vivo DRAT activity in these mutants.

When placed in a wild-type (UR2) background with the wild-type level of DRAG, a strain with DRAT-K103E (UR508) again showed lower nitrogenase activity and a higher degree of modification of dinitrogenase reductase than the wild-type control (UR506), but a strain with DRAT-N248D (UR581) showed no significant differences from the wild-type control (Table 4, Fig. 2), indicating that DRAT-N248D has lower 'constitutive' activity than that of DRAT-K103E. These results indicate that DRAT-K103E at this elevated level can compete with the normal level of DRAG to modify dinitrogenase reductase, whereas DRAT-N248D cannot.

The strongest evidence for the regulation of DRAT-WT activity in vivo is that $\mathrm{DraG}^{-}$mutants (which have functioning DRAT) showed high nitrogenase activity and had little modification of dinitrogenase reductase under $\mathrm{N}_{2}$-fixing conditions, indicating that DRAT must be post-translationally inactivated under these conditions (Liang et al., 1991; Zhang et al., 1992). To compare DRAT variants with DRAT-WT in a $\mathrm{DraG}^{-}$background, a single copy of wild-type or 'constitutively' active draT was integrated into UR472 (a draTGB deletion mutant). The nitrogenase activity in these transconjugants was monitored under $\mathrm{N}_{2}$-fixing conditions. In contrast to UR589 (DRAT-WT), in which nitrogenase activity is high (530 nmol ethylene per $\mathrm{h}$ per $\mathrm{ml}$ of cells at $\mathrm{OD}_{600} 1$ ), both UR583 (DRAT-K103E) and UR610 (DRAT-N248D) display low levels of nitrogenase activity (50 and $70 \mathrm{nmol}$ ethylene per $\mathrm{h}$ per $\mathrm{ml}$ of cells at $\mathrm{OD}_{600} 1$, respectively), reflecting the level of DRAT activity that escapes regulation in these mutants. Western blots of dinitrogenase reductase showed that whilst little modification of dinitrogenase reductase was seen in UR589 $\left(\right.$ draT $\left.^{+}\right)$, dinitrogenase reductase in both UR583 and UR610 was completely modified (Fig. 3). This result confirms the altered regulation of DRAT in these strains and this identification of such 'constitutively' active DRAT variants strongly suggests that the model of DRAT regulation through inhibition by negative effectors is correct.

Interestingly, it seems that more than one subunit of dinitrogenase reductase was modified in these mutants, especially after a shift to darkness, as more than $50 \%$ of the subunits of dinitrogenase reductase appeared to be 


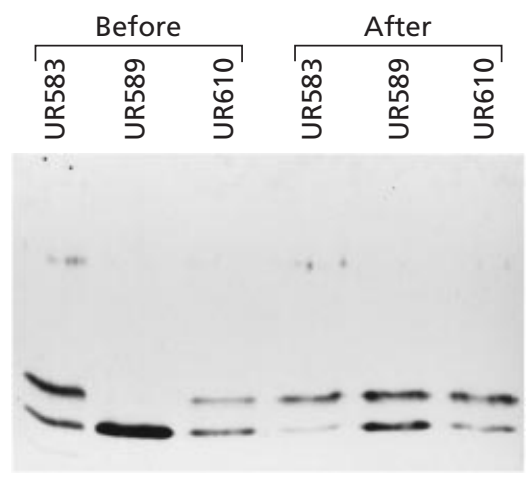

Fig. 3. ADP-ribosylation of dinitrogenase reductase in UR583 (DRAT-K103E), UR589 (DRAT-WT) and UR610 (DRAT-N248D). Each of these strains has a single copy of various $d r a T$ alleles in the chromosome, without functional draG. Crude extracts were prepared from cells before and after a shift of the cultures to darkness for $60 \mathrm{~min}$.

ADP-ribosylated. The reason for this apparent overmodification is unknown.

\section{In vitro characterization of DRAT-K103E}

Previous experiments have indicated that more DRAT protein was accumulated in the cell when $\operatorname{draTG}$ were overexpressed together (Grunwald et al., 1995). However, UR587, overexpressing both DRAT-K103E and DRAG, grew very slowly in MG medium anaerobically under light, which is probably due to a futile cycle of ADP-ribosylation, depleting the NAD pool. Instead, UR 578, in which only DRAT-K103E was expressed, was used for overexpression of DRAT-K103E, and it grew only slightly more slowly than did UR2 (wild-type). DRAT-K103E was purified according to the published protocol for DRAT-WT, with some modifications (as described in Methods), since DRAT-K103E showed substantially weaker binding to Affi-gel. The difference in affinity to Affi-gel is interesting, since Affi-gel Blue is usually used to purify proteins containing a dinucleotide fold (Thompson et al., 1975); it suggests that DRATWT and DRAT-K103E might have different abilities to bind dinucleotides.

The $K_{\mathrm{m}}$ for NAD and the $V_{\max }$ for DRAT-WT and DRAT-K103E in the presence of ADP were determined. When dinitrogenase reductase from Azotobacter vinelandii was used as an acceptor of ADP-ribose in an in vitro assay, the $V_{\max }$ (nmol ADP-ribose transferred per min per mg of protein) values of DRAT-WT and DRAT-K103E were $89 \pm 3$ and $71 \pm 2$, respectively, and the $K_{\mathrm{m}}$ values for NAD of DRAT-WT and DRAT$\mathrm{K} 103 \mathrm{E}$ were $268 \pm 31 \mu \mathrm{M}$ and $187 \pm 20 \mu \mathrm{M}$, respectively. When dinitrogenase reductase from $R$. rubrum was used as the substrate, DRAT-WT again displayed a $K_{\mathrm{m}}$ value for NAD that was about $30 \%$ higher than that of DRAT-K103E (data not shown). These results indicate that any differences in the $K_{\mathrm{m}}$ for NAD or $V_{\max }$ do not seem sufficient to explain the altered regulation in DRAT-K103E.
In vivo modification of dinitrogenase reductase in a nifD mutant by DRAT-WT and DRAT-K103E

Halbleib et al. (2000) reported recently that DRAT can modify only oxidized dinitrogenase reductase and that DRAG only removes an ADP-ribosyl group from reduced dinitrogenase reductase. In an $R$. rubrum nifD mutant, most dinitrogenase reductase is reduced, because it lacks dinitrogenase to accept electrons from it; the rate of modification of dinitrogenase reductase is very slow in this mutant (Halbleib et al., 2000). To test if DRAT-K103E responds differently to the redox states of dinitrogenase reductase, both pYPZ173 (DRATK103E) and pYPZ187 (DRAT-WT) were transferred separately into the $R$. rubrum nifD mutant and integrated into the chromosome. The rate of modification of dinitrogenase reductase was similar in both strains (data not shown), indicating that DRAT-K103E is similar to DRAT-WT in its ability to modify reduced dinitrogenase reductase.

\section{Further characterization of the mutant with DRAT-N46D (UR537)}

In preliminary screening, UR537 showed an interesting phenotype. It had substantial nitrogenase activity under derepression conditions, but responded normally to darkness, indicating that DRAT-N46D could still be activated. Western blotting of DRAT revealed that this mutant accumulated normal amounts of DRAT protein (data not shown). When a single copy of altered $d r a T$ was integrated in a DraG ${ }^{-}$background, UR619 showed substantial nitrogenase activity $(650 \mathrm{nmol}$ ethylene per $\mathrm{h}$ per $\mathrm{ml}$ of cells at $\mathrm{OD}_{600} 1$ ) and a normal darkness response (data not shown). However, in response to $\mathrm{NH}_{4}^{+}$, UR619 responded poorly and $80 \%$ residual nitrogenase activity remained after $10 \mathrm{mM} \mathrm{NH}_{4} \mathrm{Cl}$ was added for $60 \mathrm{~min}$, compared to only $10 \%$ residual nitrogenase activity remaining in the wild-type control (UR589) (data not shown). In crude extracts, UR619 had about $8 \%$ of the DRAT activity of UR589 (data not shown), indicating that $\mathrm{N} 46 \mathrm{D}$ substitution substantially reduced (but did not completely eliminate) DRAT activity. Previous data suggested that darkness generated a stronger signal for the activation of DRAT, since a faster rate of modification of dinitrogenase reductase and a lower residual nitrogenase activity were seen in response to darkness than in response to $\mathrm{NH}_{4}^{+}$(Zhang et al., 1995a). It is our hypothesis that the signal from darkness is strong enough to activate DRAT-N46D, but that the signal from $\mathrm{NH}_{4}^{+}$is too weak to do so.

\section{DISCUSSION}

Although there is substantial evidence that both DRAT and DRAG activities themselves are regulated, the mechanism for this regulation is unknown. Two general hypotheses have been proposed for the nature of DRAT and DRAG regulation: (1) regulation through interaction with the substrate, dinitrogenase reductase, and (2) regulation through interaction with either small molecules or other protein effectors. 
It is clear that dinitrogenase reductase is involved in some portion of the regulation of DRAT and DRAG activities. The most striking case is the recent demonstration that the different redox states of dinitrogenase reductase are required for DRAT and DRAG (Halbleib et al., 2000). DRAT can modify only oxidized dinitrogenase reductase and DRAG removes the ADPribosyl group only from reduced dinitrogenase reductase. The second indication that substrate recognition affects DRAT activity comes from the results of Kim et al. (1999), who employed a completely different genetic hunt for DRAT variants that were able to modify an E112K variant of dinitrogenase reductase. This E112K variant of dinitrogenase reductase is a poor substrate for wild-type DRAT. Surprisingly, this different search also identified DRAT-K103E, as well as another DRAT-Q81R variant. Further analysis showed that DRAT-Q81R had a lower level of 'constitutive' DRAT activity than DRAT-K103E and DRAT-N248D. In a complementary study, DRAT-N248D, identified in the present screen for altered regulation, was also able to modify the E112K variant of dinitrogenase reductase (Kim et al., 1999). These results suggest that there is a correlation between the regulation of DRAT activity and its substrate recognition.

Despite the importance of dinitrogenase reductase in the regulation of DRAT/DRAG activities, this cannot be the entire basis of the regulation, for four reasons. (1) As demonstrated in this work, the 'constitutively' active DRAT (DRAT-K103E) is not altered in its ability to ADP-ribosylate reduced dinitrogenase reductase. (2) The degree of the redox effect on DRAT/DRAG does not seem to be sufficient to account for the extremely tight regulation of DRAT/DRAG seen in vivo. Little DRAT activity was found under nitrogen-limiting conditions, but the redox states of dinitrogenase reductase change continuously during substrate reduction. (3) DRAT and DRAG are not always regulated in a coordinated way and both proteins can be inactive in some conditions, so the regulation of DRAT and DRAG must involve more complicated or totally different mechanisms. Consistent with this, only the regulation of DRAG appears to be altered in R. rubrum and $A$. brasilense ntrBC mutants, but DRAT regulation is altered in an R. rubrum glnB mutant (Zhang et al., 1994, 1995b, 2000). (4) Effects of $n t r B C$ mutations on the regulation of DRAG activity in R. rubrum are extremely difficult to trace to effects on the substrate itself, since it has no significant effect on nif expression (Zhang et al., 1994, 1995b). It is more likely that this mutation perturbs either small molecules or protein effectors which interact with DRAG in vivo.

The different affinity to the Affi-gel Blue column of DRAT-WT and DRAT-K103E is extremely interesting, as it suggests that the basis for the altered regulation of this DRAT variant might be due to a different affinity for a dinucleotide or a similar molecule. The regulation of DRAT activity through its accessibility to NAD would be a very attractive hypothesis, based on the fact that NAD is required for the interaction between DRAT and dinitrogenase reductase in vitro (Grunwald \& Ludden, 1997) and on reports showing that exogenous NAD can perturb the modification of dinitrogenase reductase (Norén et al., 1997; Soliman \& Nordlund, 1992). However, our in vitro analysis of the affinity for NAD of DRAT-WT and DRAT-K103E would seem to preclude this as the basis for the altered regulation by DRAT variants, suggesting that NAD alone is not the direct effector for regulation of DRAT in vivo. Nevertheless, the dramatically altered affinity of DRAT-K103E to the Affi-gel Blue column, compared to that of DRAT-WT, suggests a significant alteration in the surface of this DRAT variant and is consistent with altered binding either to some small molecules or to protein effectors.

In summary, the data in this report strongly suggest that DRAT activity is regulated in vivo, probably via a small molecule effector such as a nucleotide or a dinucleotide. Whilst identification of such an effector might be difficult, we believe that the DRAT variants described here will prove to be a highly valuable control in screening for these effectors with the in vitro DRAT assay.

\section{ACKNOWLEDGEMENTS}

This work was supported by the College of Agricultural and Life Sciences, University of Wisconsin-Madison, Department of Agriculture grant 99-35305-8010 to G.P.R. and NIGMS grant 54910 to P. W.L. We thank S. K. Grunwald, V. K. Shah, Y. Ma and E. L. Pohlmann for technical help and advice.

\section{REFERENCES}

Ditta, G., Schmidhauser, T., Yakobson, E., Lu, P., Liang, X., Finlay, D. R., Guiney, D. \& Helinski, D. R. (1985). Plasmids related to the broad range vector, pRK290, useful for gene cloning and for monitoring gene expression. Plasmid 13, 149-153.

Fitzmaurice, W. P., Saari, L. L., Lowery, R. G., Ludden, P. W. \& Roberts, G. P. (1989). Genes coding for the reversible ADPribosylation system of dinitrogenase reductase from Rhodospirillum rubrum. Mol Gen Genet 218, 340-347.

Fu, H. A., Hartmann, A., Lowery, R. G., Fitzmaurice, W. P., Roberts, G. P. \& Burris, R. H. (1989). Posttranslational regulatory system for nitrogenase activity in Azospirillum spp. J Bacteriol 171, 4679-4685.

Fu, H. A., Fitzmaurice, W. P., Roberts, G. P. \& Burris, R. H. (1990). Cloning and expression of draTG genes from Azospirillum lipoferum. Gene 86, 95-98.

Grunwald, S. K. \& Ludden, P. W. (1997). NAD-dependent crosslinking of dinitrogenase reductase and dinitrogenase reductase ADP-ribosyltransferase from Rhodospirillum rubrum. J Bacteriol 179, 3277-3283.

Grunwald, S. K., Lies, D. P., Roberts, G. P. \& Ludden, P. W. (1995). Posttranslational regulation of nitrogenase in Rhodospirillum rubrum strains overexpressing the regulatory enzymes dinitrogenase reductase ADP-ribosyltransferase and dinitrogenase reductase activating glycohydrolase. J Bacteriol 177, 628-635.

Halbleib, C. M., Zhang, Y. \& Ludden, P. W. (2000). Regulation of dinitrogenase reductase ADP-ribosyltransferase and dinitrogenase reductase-activating glycohydrolase by a redox-dependent conformational change of nitrogenase Fe protein. J Biol Chem 275, 3493-3500. 
Hartmann, A. \& Burris, R. H. (1987). Regulation of nitrogenase activity by oxygen in Azospirillum brasilense and Azospirillum lipoferum. J Bacteriol 169, 944-948.

Hartmann, A., Fu, H. \& Burris, R. H. (1986). Regulation of nitrogenase activity by ammonium chloride in Azospirillum spp. J Bacteriol 165, 864-870.

Inoue, A., Shigematsu, T., Hidaka, M., Masaki, H. \& Uozumi, T. (1996). Cloning, sequencing and transcriptional regulation of the draT and draG genes of Azospirillum lipoferum FS. Gene 170, 101-106.

Jouanneau, Y., Roby, C., Meyer, C. \& Vignais, P. M. (1989). ADP-ribosylation of dinitrogenase reductase in Rhodobacter capsulatus. Biochemistry 28, 6524-6530.

Kanemoto, R. H. \& Ludden, P. W. (1984). Effect of ammonia, darkness, and phenazine methosulfate on whole-cell nitrogenase activity and Fe protein modification in Rhodospirillum rubrum. J Bacteriol 158, 713-720.

Keohavong, P. \& Thilly, W. G. (1989). Fidelity of DNA polymerases in DNA amplification. Proc Natl Acad Sci USA 86, 9253-9257.

Kim, K., Zhang, Y. \& Roberts, G. P. (1999). Correlation of activity regulation and substrate recognition of the ADP-ribosyltransferase that regulates nitrogenase activity in Rhodospirillum rubrum. J Bacteriol 181, 1698-1702.

Lehman, L. J. \& Roberts, G. P. (1991). Identification of an alternative nitrogenase system in Rhodospirillum rubrum. J Bacteriol 173, 5705-5711.

Leung, D. W., Chen, E. \& Goedde, D. V. (1989). A method for random mutagenesis of a defined DNA segment using a modified polymerase chain reaction. Technique 1, 11-15.

Liang, J. H., Nielsen, G. M., Lies, D. P., Burris, R. H., Roberts, G. P. \& Ludden, P. W. (1991). Mutations in the $d r a T$ and $d r a G$ genes of Rhodospirillum rubrum result in loss of regulation of nitrogenase by reversible ADP-ribosylation. J Bacteriol 173, 6903-6909.

Lowery, R. G. \& Ludden, P. W. (1988). Purification and properties of dinitrogenase reductase ADP-ribosyltransferase from the photosynthetic bacterium Rhodospirillum rubrum. J Biol Chem 263, 16714-16719.

Lowery, R. G. \& Ludden, P. W. (1989). Effect of nucleotides on the activity of dinitrogenase reductase ADP-ribosyltransferase from Rhodospirillum rubrum. Biochemistry 28, 4956-4961.

Ludden, P. W. \& Roberts, G. P. (1989). Regulation of nitrogenase activity by reversible ADP ribosylation. Curr Top Cell Regul 30, 23-56.

Lundberg, K. S., Shoemaker, D. D., Adams, M. W. W., Short, J. M., Sorge, J. A. \& Mathur, E. J. (1991). High-fidelity amplification using a thermostable DNA polymerase isolated from Pyrococcus furiosus. Gene 108, 1-6.

Ma, L. \& Li, J. (1997). Cloning and sequencing of draTG genes and their downstream region of Azospirillum brasilense Yu62. Chin J Biotechnol 13, 143-152.

Masepohl, B., Krey, R. \& Klipp, W. (1993). The draTG gene region of Rhodobacter capsulatus is required for post-translational regulation of both the molybdenum and the alternative nitrogenase. J Gen Microbiol 139, 2667-2675.

Norén, A., Soliman, A. \& Nordlund, S. (1997). The role of NAD as a signal during nitrogenase switch-off in Rhodospirillum rubrum. Biochem J 322, 829-832.
Pierrard, J., Ludden, P. W. \& Roberts, G. P. (1993). Posttranslational regulation of nitrogenase in Rhodobacter capsulatus: existence of two independent regulatory effects of ammonium. J Bacteriol 175, 1358-1366.

Schweizer, H. P. (1993). Small broad-host-range gentamycin resistance gene cassettes for site-specific insertion and deletion mutagenesis. BioTechniques 15, 831-833.

Simon, R., Priefer, U. \& Pühler, A. (1983). A broad host range mobilization system for in vivo genetic engineering: transposon mutagenesis in Gram negative bacteria. Bio/Technology 1, 784-791.

Soliman, A. \& Nordlund, S. (1992). Studies on the effect of $\mathrm{NAD}(\mathrm{H})$ on nitrogenase activity in Rhodospirillum rubrum. Arch Microbiol 157, 431-435.

Thompson, S. T., Cass, K. H. \& Stellwagen, E. (1975). Blue Dextran-sepharose: an affinity column for the dinucleotide fold in protein. Proc Natl Acad Sci US A 72, 669-672.

Triplett, E. W., Wall, J. D. \& Ludden, P. W. (1982). Expression of the activating enzyme and $\mathrm{Fe}$ protein of nitrogenase from Rhodospirillum rubrum. J Bacteriol 152, 786-791.

Vieira, J. \& Messing, J. (1982). The pUC plasmids, an M13mp7derived system for insertion mutagenesis and sequencing with synthetic universal primers. Gene 19, 259-268.

Vogel, A. M. \& Das, A. (1994). Mutational analysis of Agrobacterium tumefaciens pTiA6 virD1: identification of functionally important residues. Mol Microbiol 12, 811-817.

Zhang, Y., Burris, R. H. \& Roberts, G. P. (1992). Cloning, sequencing, mutagenesis, and functional characterization of $d r a T$ and draG genes from Azospirillum brasilense. J Bacteriol 174, 3364-3369.

Zhang, Y., Burris, R. H., Ludden, P. W. \& Roberts, G. P. (1993). Posttranslational regulation of nitrogenase activity by anaerobiosis and ammonium in Azospirillum brasilense. J Bacteriol 175, 6781-6788.

Zhang, Y., Burris, R. H., Ludden, P. W. \& Roberts, G. P. (1994). Posttranslational regulation of nitrogenase activity in Azospirillum brasilense ntrBC mutants: ammonium and anaerobic switch-off occurs through independent signal transduction pathways. J Bacteriol 176, 5780-5787.

Zhang, Y., Burris, R. H., Ludden, P. W. \& Roberts, G. P. (1995a). Comparison studies of dinitrogenase reductase ADP-ribosyl transferase/dinitrogenase reductase activating glycohydrolase regulatory systems in Rhodospirillum rubrum and Azospirillum brasilense. J Bacteriol 177, 2354-2359.

Zhang, Y., Cummings, A. D., Burris, R. H., Ludden, P. W. \& Roberts, G. P. (1995b). Effect of an $n t r B C$ mutation on the posttranslational regulation of nitrogenase activity in Rhodospirillum rubrum. J Bacteriol 177, 5322-5326.

Zhang, Y., Burris, R. H., Ludden, P. W. \& Roberts, G. P. (1997). Regulation of nitrogen fixation in Azospirillum brasilense. FEMS Microbiol Lett 152, 195-204.

Zhang, Y., Pohlmann, E. L., Ludden, P. W. \& Roberts, G. P. (2000). Mutagenesis and functional characterization of the $g \ln B, g \ln A$, and nifA genes from the photosynthetic bacterium Rhodospirillum rubrum. J Bacteriol 182, 983-992.

Received 12 June 2000; revised 6 September 2000; accepted 13 September 2000. 